\title{
Optimization of soft X-ray tomography on the COMPASS tokamak
}

\author{
Martin Imríšek, \\ Jan Mlynár̆, \\ Viktor Löffelmann, \\ Vladimír Weinzettl, \\ Tomáš Odstrčil, \\ Michal Odstrčil, \\ Matěj Tomeš
}

\begin{abstract}
The COMPASS tokamak is equipped with the soft X-ray (SXR) diagnostic system based on silicon photodiode arrays shielded by a thin beryllium foil. The diagnostic is composed of two pinhole cameras having 35 channels each and one vertical pinhole camera with 20 channels, which was installed recently to improve tomographic inversions. Lines of sight of the SXR detectors cover almost complete poloidal cross section of the COMPASS vessel with a spatial resolution of 1-2 cm and temporal resolution of about $3 \mu \mathrm{s}$. Local emissivity is reconstructed via Tikhonov regularization constrained by minimum Fisher information that provides reliable and robust solution despite limited number of projections and ill-conditionality of this task. Improved border conditions and numerical differentiation matrices suppressing artifacts in reconstructed radiation were implemented in the code. Furthermore, a fast algorithm eliminating iterative processes was developed, and it is foreseen to be tested in real-time plasma control.
\end{abstract}

Key words: soft X-ray $\bullet$ tomography $\bullet$ Tikhonov regularization $\bullet$ tokamak

M. Imríšek ${ }^{\bowtie}$ J. Mlynář, V. Löffelmann, V. Weinzettl, M. Tomeš

Institute of Plasma Physics ASCR v.v.i.,

Faculty of Mathematics and Physics,

Charles University in Prague,

Za Slovankou 3, 18200 Praha 8, Czech Republic,

Tel.: +42060 3810107 ,

E-mail: imrisek@ipp.cas.cz

T. Odstrčil

Max-Planck-Institut für Plasmaphysik,

Technische Universität Munchen,

Boltzmannstraße 2, D-85748 Garching, Germany

\section{Odstrčil}

Optoelectronic Research Center,

University of Southampton,

University Rd, Southampton SO17 1BJ, United Kingdom

Received: 3 September 2015

Accepted: 11 November 2015

\section{Introduction}

In tokamak plasmas, soft X-ray (SXR) radiation represents a useful quantity for diagnostics of various processes in plasma core. SXR radiation power is given predominantly by bremsstrahlung and line radiation of heavy impurities (tungsten, iron). Bremsstrahlung is generated mainly by the elastic electron-ion collisions (in the nonrelativistic limit, radiation fields produced by two particles in like-particle collision cancel) and the SXR-radiated power density is, therefore, dependent on electron density $n_{\mathrm{e}}$, density of $\mathrm{Z}_{j}$-times charged ion species $n_{j}$, and electron temperature $T_{\mathrm{e}}$ :

$$
P_{\text {brem }} \propto \sum_{j} z_{j}^{2} n_{j} n_{\mathrm{e}} \sqrt{T_{\mathrm{e}}}
$$

Line radiation is emitted by bound-bound transitions of excited electrons in atoms and non-fully ionized ions. In the case of negligible particle transport, intensity of the line radiation from specific location is proportional to the density of ion species, electrons, and it is also a function of electron temperature, as collisions with free electrons excite bound electrons. In tokamaks, SXR line radiation is generated by heavy impurities such as tungsten or iron, which can contaminate plasma because of plasma wall interactions. Impurity accumulation has a serious impact on the tokamak performance, as it results in a cooling of plasma and a dilution of the hydrogen isotopes (i.e., reduction of possible fusion reactions rate). 
The character of mechanisms generating the SXR radiation makes its measurement suitable for monitoring plasma processes connected with perturbation of density and temperature (sawtooth instability, magnetohydrodynamic (MHD) modes) and monitoring impurity profiles with high temporal and spatial resolution. Moreover, SXR measurements can provide information on plasma position and shape.

\section{Soft X-ray diagnostic on the COMPASS tokamak}

The COMPASS tokamak is a divertor device with clear H-mode and ITER-relevant geometry (1:10 to ITER plasma size, $R=0.56 \mathrm{~m}, a=0.23 \mathrm{~m}, I_{p}<400 \mathrm{kA}$, $\mathrm{B}_{\mathrm{T}} \sim 1.15 \mathrm{~T}$, and a typical pulse length of about $300 \mathrm{~ms}$ ) [1]. Limiter and divertor tiles are made of graphite. Therefore, the concentration of heavy impurities in plasma is low and the main source of the SXR radiation is bremsstrahlung.

The current SXR diagnostic system at COMPASS consists of three pinhole cameras. Each camera has one photodiode array shielded by a $10-\mu \mathrm{m}$ thick beryllium foil. The system contains two arrays of 35 detectors (type LD35-5T) and one array of 20 detectors [2] (IRD, type AXUV-20EL) covering almost the whole poloidal cross section (see Fig. 1 and Fig. 2). The spectral sensitivity (shown in Fig. 3) is given mainly by the thickness of the beryllium foil blocking low energy photons (below $1 \mathrm{keV}$ ) and by the diode active layer, which is $200 \mu \mathrm{m}$ thick. Temporal resolution is limited mainly by the amplifier to $3 \mu \mathrm{s}$, allowing investigation of fast MHD processes such as internal kink modes (with frequency of about $40 \mathrm{kHz}$ ) or sawtooth oscillations (about $500 \mathrm{~Hz}$ ). The spatial resolution of each camera is $1-2 \mathrm{~cm}$.

\section{Tomographic reconstruction}

Tomography allows reconstructing local parameters from line-integrated measurements. Its solution can be found either analytically by transformation

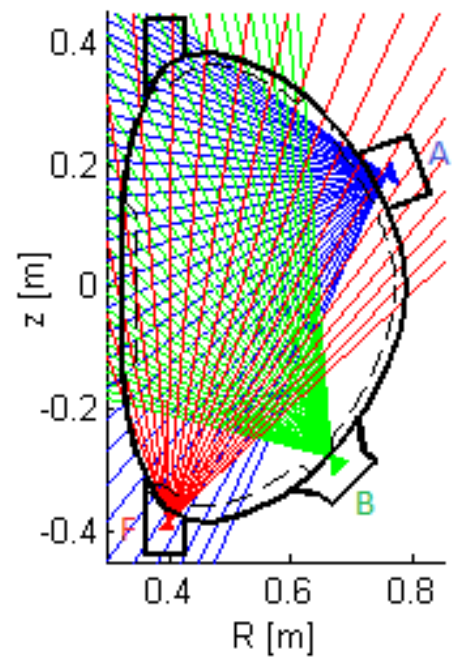

Fig. 1. Lines of sight of the SXR detectors on the COMPASS tokamak.

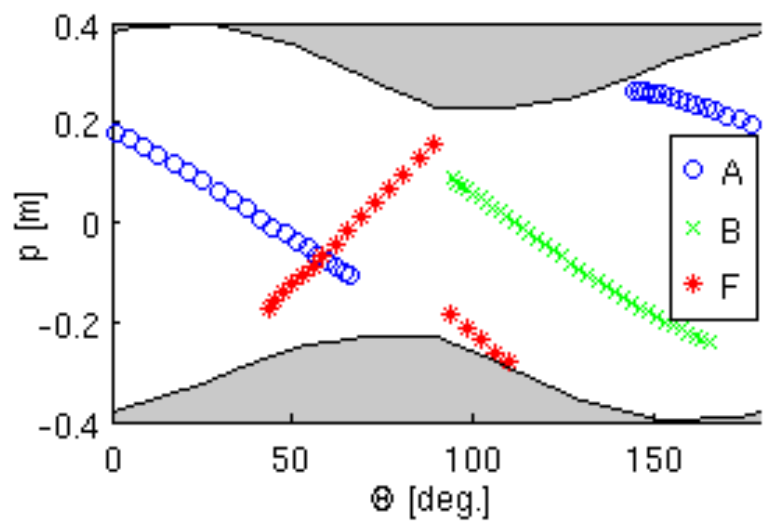

Fig. 2. Coverage in projection space where $p$ is the distance from the center of chamber and $\Theta$ angle with respect to the horizontal axis.

methods or by series expansion methods searching for a solution given as a linear combination of basis functions, which can be global (harmonics, Zernike polynomials) or local (pixels). In fusion research, plasma emissivity is usually reconstructed by series expansion methods because transformation methods are effective only for regular and dense projections of the reconstructed area, which is not satisfied in tokamaks because of engineering constraints. Furthermore, series expansion methods allow easier implementation of a priori information. Series expansion methods are preferably used with local basis functions, in particular, with rectangular pixels, because the alternative approach using global basis functions usually requires a precise knowledge of the plasma center position. In the case of pixel methods, the reconstruction is performed by inversion of the following system of equations:

$$
f_{i}=\sum_{j}^{N} T_{i j} g_{j}+\zeta_{i}
$$

where $f_{i}$ is the measured line-integrated radiation, $g_{j}$ the emissivity associated with the particular pixel, $T_{i j}$ the contribution matrix, $N$ number of pixels, and $\zeta_{i}$ the statistical errors. Value of each element of the contribution matrix $T_{i j}$ (representing influence of the $j$-th pixel on the $i$-th detector) can be calculated as a distance which line of sight of $i$-th detector passes through $j$-th pixel. This is possible because of the fact that the intensity of a source decreases with squared distance and a collection area of each detector increases with squared distance.

The system of equations is usually highly underdetermined (on COMPASS, number of pixels is typically by two orders of magnitude larger than number of detectors), inversion represents an ill-conditioned

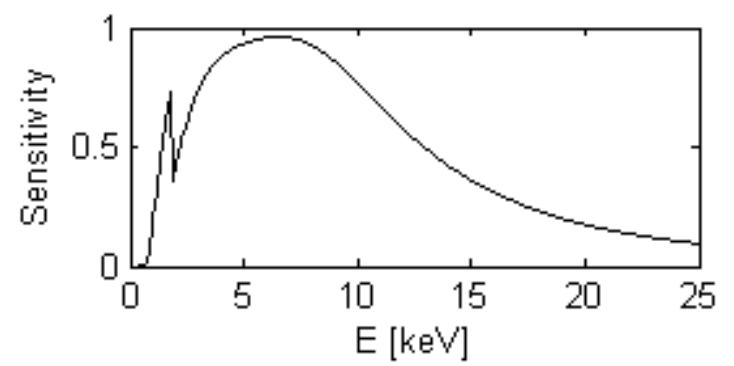

Fig. 3. Theoretical spectral sensitivity of the SXR detectors. 
task, and a noise level in the tokamak environment is high. Therefore, regularization algorithms constrained by $a$ priori information (e.g., expected smoothness) are often applied to find a unique and reliable solution. Regularization principle is close to the Occam's razor principle. From the infinite number of solutions, the one which is smooth and physically meaningful is chosen. The solution is found by minimizing

$$
\Lambda=\frac{1}{2} \chi+\alpha_{R} R
$$

where $\chi^{2}=\left\|\left(f-T_{g}\right) / \sigma_{s}\right\|$ is the goodness-of-fit parameter, $\sigma_{s}$ the expected data error bars, $R$ regularizing functional, and $\alpha_{R}$ is the regularization (smoothing) parameter that represents weighting between the goodness of fit and the requirements imposed on the solution $g$ by the functional $R$.

Minimization $(d \Lambda / d g=0)$ of $\Lambda(g)$ leads to

$$
g(\alpha)=\left(\mathbf{T}^{\mathbf{T}} \mathbf{T}+\alpha \mathbf{H}\right)^{-1} \mathbf{T}^{\mathbf{T}} \mathbf{f}
$$

where $\mathbf{H}$ contains operators that calculate regularizing functional $R$ from $g: R=\mathbf{g}^{\mathrm{T}} \mathbf{H g}$. For example, a smooth solution can be found if $R$ measures 'roughness'. One of the possible choices is the operator of the first derivatives: $\mathrm{H}=\nabla_{x}^{T} * \nabla_{x}+\nabla_{y}^{T} * \nabla_{y}=\mathbf{B}_{\mathbf{x}}^{\mathrm{T}} \mathbf{B}_{\mathbf{x}}$ $+\mathbf{B}_{y}^{\mathrm{T}} \mathbf{B}_{\mathrm{y}}$, where $\mathbf{B}_{\mathrm{x}}, \mathbf{B}_{\mathbf{y}}$ correspond to matrices representing differentiation in horizontal and vertical directions, respectively.

On the COMPASS tokamak, the Fisher information is preferably applied as regularization functional to reconstruct local emissivity. The Fisher information of the probability distribution is defined by $I_{\mathrm{F}}=\int(\nabla g)^{2} / g d S$. In the statistics, the Fisher information is used to measure the amount of information that observable variable carries about the parameter upon which the probability depends. The Fisher information can be used to measure a degree of disorder of the system. In contrast to entropy, the Fisher information includes a local measure (rearrangement of points in the formula for entropy: $S=-\Sigma_{j} g_{j} \ln \left(g_{j}\right)$ does not change its value). It is related to variance of the probability distribution $\sigma$ by the Cramer-Rao inequality:

$$
\sigma^{2} \leq 1 / I_{\mathrm{F}}
$$

Thus, minimization of the Fisher information increases a lower limit of variance of the probability distribution (which is connected with an increase in entropy). In practice, this principle leads to higher smoothness in low emissivity regions. The regularization constrained by minimum Fisher information is complicated by its nonlinearity. However, the system of equations can be linearized by several iterative steps of the weighted linear regularization:

$$
\begin{aligned}
g^{(n+1)}(\alpha)= & \left(\mathbf{T}^{\mathrm{T}} \mathbf{T}+\alpha\left(\mathbf{B}_{\mathrm{x}}^{\mathbf{T}} \mathbf{W}^{(\mathrm{n})} \mathbf{B}_{\mathbf{x}}+\mathbf{B}_{\mathbf{y}}^{\mathbf{T}} \mathbf{W}^{(\mathbf{n})} \mathbf{B}_{\mathbf{y}}\right)\right)^{-1} \\
& \cdot \mathbf{T}^{\mathrm{T}} \mathbf{f}
\end{aligned}
$$

where $W_{i j}^{n}=\delta_{i j} / g_{i}^{n}$ for $n>1$ and $W^{1}=1$ for $n=$ 1 . This approach was proven to provide robust and reliable solution [3-5].
In plasma tomography, it is beneficial to include magnetic topology because any change in plasma parameters is usually much lower along the magnetic field lines. Equation (6) is then modified into the form:

$$
g^{(n+1)}(\alpha)=\left(\mathbf{T}^{\mathrm{T}} \mathbf{T}+\alpha\left(\begin{array}{l}
\exp (\eta) \mathbf{B}_{\|}^{\mathrm{T}} \mathbf{W}^{(\mathrm{n})} \mathbf{B}_{\|} \\
+\exp (-\eta) \mathbf{B}_{\perp}^{\mathrm{T}} \mathbf{W}^{(\mathrm{n})} \mathbf{B}_{\perp}
\end{array}\right)\right)^{-1} \cdot \mathbf{T}^{\mathrm{T}} \mathbf{f}
$$

where $\mathbf{B}_{\|}, \mathbf{B}_{\perp}$ correspond to numerical differentiation matrices acting parallel and perpendicular to the magnetic flux surfaces and $\eta>0$ leads to the preferential smoothing along magnetic field lines.

The regularization task has to implement a suitable choice of the regularization parameter $\alpha$. One of the methods to determine $\alpha$ is to iteratively change its value so that it matches $\chi^{2}(\alpha) \rightarrow N$, where $N$ is the number of detectors. In the algorithm, the regularization constrained by minimum Fisher information is performed in two nested loops. The outer loop minimizes the Fisher information by solving $g^{(n+1)}(\alpha)$ with $\mathbf{W}=\operatorname{diag}\left(1 / g^{(n)}\right)$ and the inner loop iteratively changes $\alpha$. Two or three cycles of the outer loop and up to 10 cycles of the inner loop usually lead to the solution with a sufficient convergence (on COMPASS, the condition $\left|\chi^{2}(\alpha)-N\right| / N<0.05$ is applied).

\section{Differentiation}

Tikhonov regularization constrained by minimum Fisher information requires calculating numerical differentiation matrices. There are several ways how to apply numerical differentiation: forward difference, $\left(g_{k+1}-g_{k}\right) / d x$; backward difference, $\left(g_{k-1}-g_{k}\right) /$ $d x$; central difference, $\left(g_{k+1}-g_{k-1}\right) / 2 d x$; and higher-order methods. Forward and backward differences are not symmetrical and can lead to artifacts, particularly in case of low resolution. Central difference is symmetrical and approximates the derivative more accurately (error is proportional to $d x^{2}$ instead of $d x$ for forward and backward difference), but it is susceptible to $2 d x$ oscillations (solution with $2 d x$ oscillation can have zero central difference) so it usually results in chessboard-like pattern. Higher-order methods decrease resolution, as they involve more pixels for the calculation of difference at particular pixel. However, the Fisher information calculated by forward or backward difference can be symmetrized by weight matrix $\mathbf{W}$ containing interpolated values of the solution between pixels:

$$
I_{\mathrm{F}}^{\text {forward }}=\sum_{k}\left(\frac{g_{k+1}-g_{k}}{d x}\right)^{2} \cdot \frac{2}{g_{k}+g_{k+1}}
$$

In general, the weight matrix can be written in the form $w_{i i^{(n+1)}}=\delta_{i j} /\left(|\mathbf{B}| g^{(n)}\right)_{i}$. In the case of classical smoothing along vertical and horizontal axis, calculations of the Fisher information by forward differentiation and backward differentiation are equivalent. In the case of preferential smoothing along magnetic field lines, the algorithm finds more precise solution when the Fisher information calculated by forward and backward differentiation are combined: 


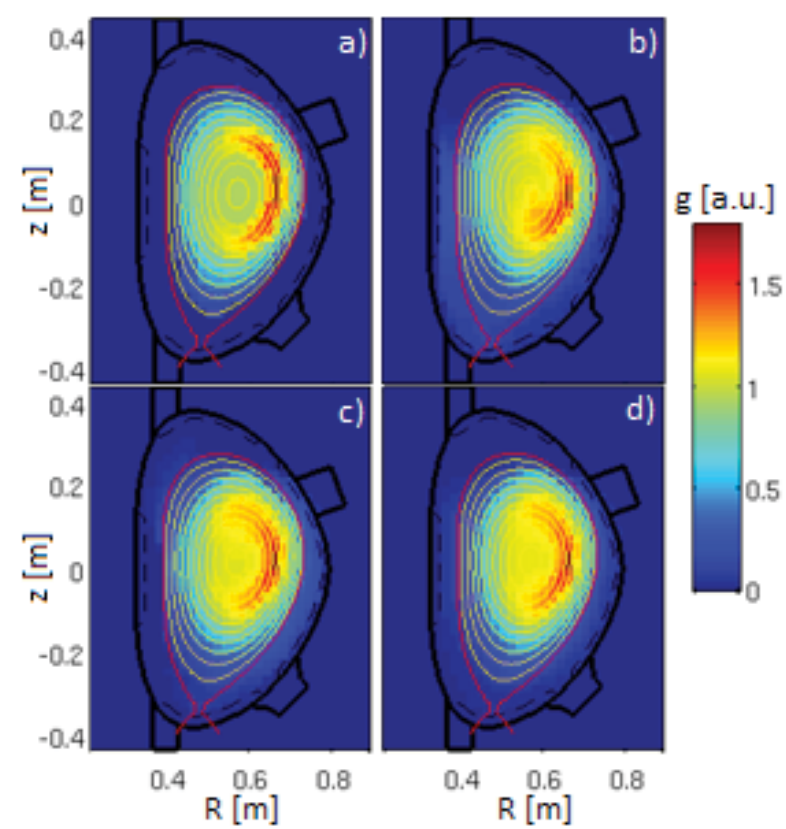

Fig. 4. Model function (a), reconstruction using forward (clockwise) differentiation (b), backward (anticlockwise) differentiation (c), and combination of forward and backward differentiation $(\mathrm{d})$.

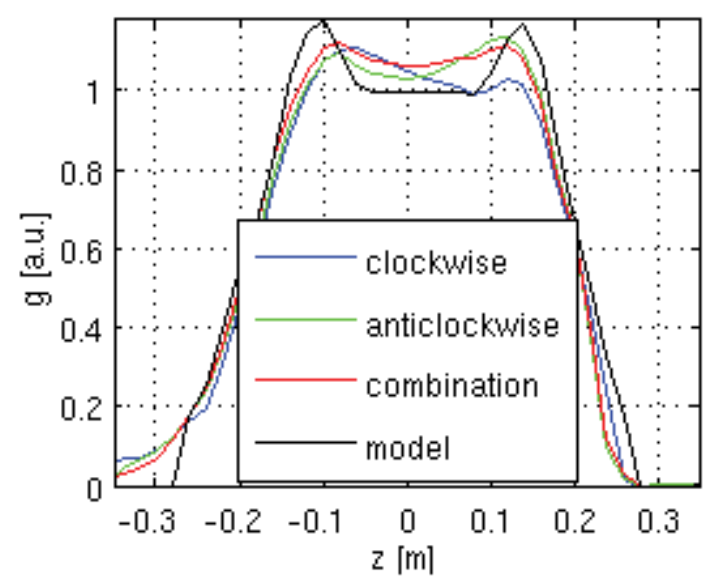

Fig. 5. Vertical profile of the model function and the reconstructions using forward (clockwise) difference, backward difference (anticlockwise), and their combination at $R=0.56 \mathrm{~m}$.

$$
I_{\mathrm{F}}=\frac{1}{2} I_{\mathrm{F}}^{\text {forward }}+\frac{1}{2} I_{\mathrm{F}}^{\text {backward }}
$$

As shown in Figs. 4 and 5, applications of different calculations of the Fisher information for reconstruction of phantom function modelling banana profile of emissivity because of the accumulation of impurities and toroidal plasma rotation are compared. Combination of the forward and backward difference led to lower deviation from the model (5\%) compared to forward $(6.4 \%)$ or backward $(8.4 \%)$ difference. The discrepancy between model agreement for a forward and backward difference is probably caused by different values of the Fisher information in the case of varying direction of differentiation (i.e., in the case of magnetic field lines). In the case of standard differentiation (in vertical and horizontal direction), the Fisher information calcu- lated by the formula (8) is the same for forward and backward difference (with zero border condition).

\section{Border conditions}

Border conditions are particularly important when significant emissivity is to be reconstructed near the border that is usually not the case of SXR tomography. However, it can be worthy to restrict the reconstructed SXR emissivity by border conditions, as they can favourably affect the whole profile. Reconstructed emissivity should be zero outside the tokamak vessel. In order to meet this condition, the algorithm excludes pixels outside the chamber from the calculation and then imposes zero emissivity requirement on chamber's pixels. Exclusion of pixels outside the chamber leads to a faster reconstruction because the computational time increases with the number of pixels (matrix inversion is computed via the Cholesky decomposition whose computational time is proportional to the cubed number of pixels). Requirement of zero emissivity at chamber's pixels can be implemented by two ways: by matrix operator for the Fisher information or by contribution matrix. The former represents smoothing of the edge under the condition of zero values at the chamber, and the later acts as an extension of the detector system by virtual detectors observing zero signals from pixels at the chamber (in the present implementation, each detector sees one pixel at the chamber). Figure 6 compares reconstructed profiles of the model function for different border conditions. In the case of no border conditions, the reconstruction of used model (Gaussian) function led to overestimation of emissivity in the edge of plasma and a higher error in the core. The precision of the reconstruction with border conditions depends on the shape of reconstructed profile. For example, in the case of
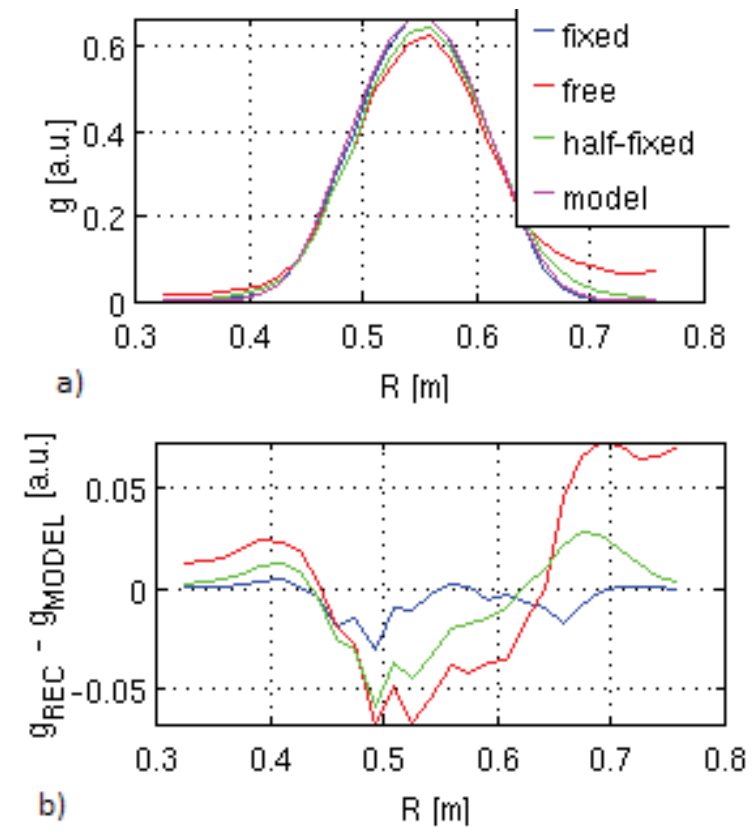

Fig. 6. Profiles reconstructed with different constraint on border conditions for model function $g \sim \exp \left(-r^{3}\right)$ (a) and their differences from the model function (b). 

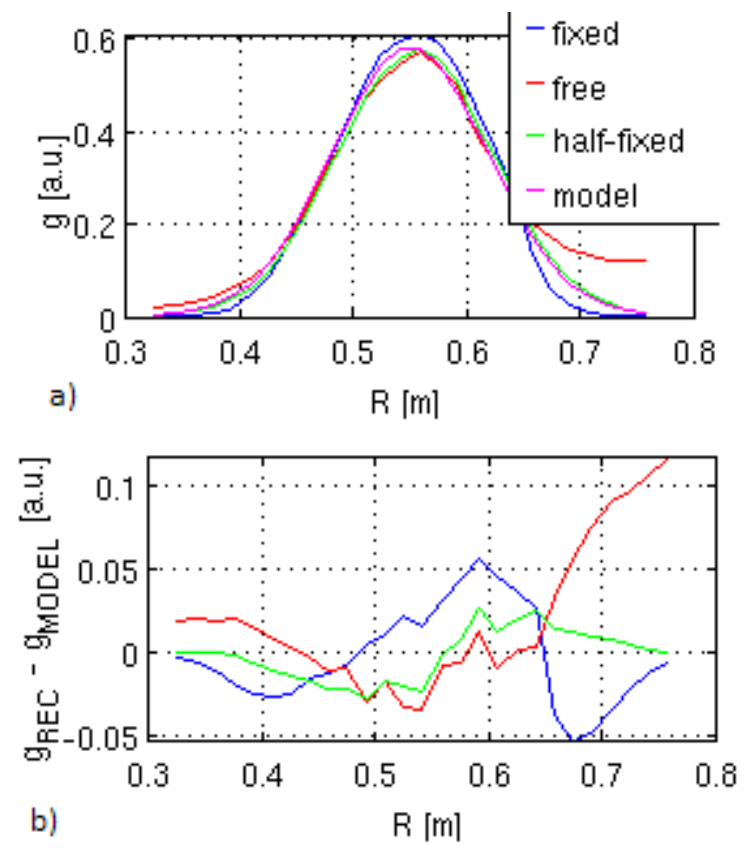

Fig. 7. Profiles reconstructed with different constraint on border conditions for model function $g \sim \exp \left(-r^{2}\right)$ (a) and their differences from the model function (b).

model function shown in Fig. 7a $\left(g \sim \exp \left(-r^{3}\right)\right.$ where $r$ represents the distance from the center), the most accurate reconstruction uses 'half-fixed' borders, that is, reconstruction with lower weight (lower values representing border condition in the matrix operator) of border conditions on the solution. As the SXR profile measured in the COMPASS tokamak is usually highly peaked and located in the plasma center, the fixed border conditions are applied.

\section{Rolling iteration}

The plasma radiation profile can be assumed to be evolving smoothly during most phases of the discharge. Using this assumption, the iteration can be accelerated by using the results from the last time frame as initial guesses for the current frame (both the values of $\alpha$ and $g$ ). Alternatively, this may be viewed as doing the iteration continuously while changing the input data at certain moments (denoted as rolling iteration). The result is returned at the same time.

An extreme form of the rolling iteration is changing the data in every iteration step. In this case, the iteration is left no time to converge when the initial guess is inaccurate. The quality of the solution depends on the assumption of smooth plasma evolution. However, the long-term stability of the method remains good. In the case of a sudden change in the emissivity profile, it typically takes several frames for the method to approach the correct solution again. The convergence rate differs for various quantities estimated from the reconstructions. For example, the error of plasma position seems to decay exponentially with a time constant of 3-4 frames. In contrast, the changes in total radiated power are reconstructed instantly [6].

For the tomographic algorithms with linear regularization functional, the rolling iteration reduces to
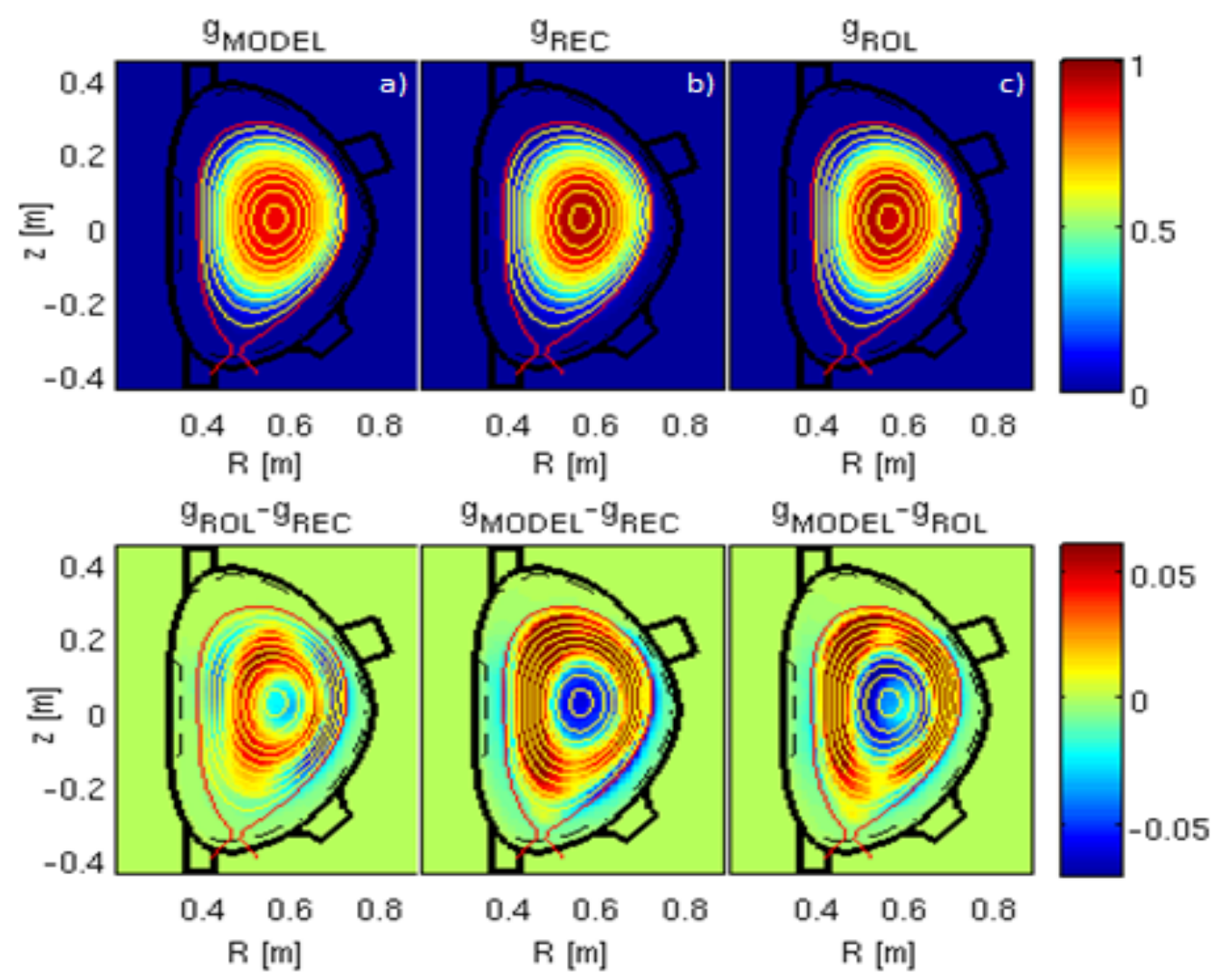

Fig. 8. Model function $g_{\text {model }}(\mathrm{a})$, its tomographic reconstruction $g_{\text {REC }}\left(\right.$ b), reconstruction by the rolling iteration $g_{\text {ROL }}$ (c) just after a modelled sawtooth crash (sudden flattening of the profile), and their differences. 
a fast and less accurate way to estimate $\alpha$. However, there is a possibility to exploit the knowledge of previous result more. The system of linear equations can be solved using an iterative method, which needs an initial approximation. The previous result may be used as this approximation, which may reduce the number of iterations that is needed to reach the required accuracy. This approach is currently under development on COMPASS.

Figure 8 shows phantom function modelling sawtooth oscillations just after the sawtooth crash (sudden flattening of the profile) and its tomographic reconstruction by standard algorithm and rolling iteration. The differences between the model function and both reconstructions show an overestimation of emissivity in the center, which is a typical behaviour of the Tikhonov regularization constrained by minimum Fisher information in the case of profiles with low peakedness [7]. The difference between the standard algorithm and the rolling iteration is lower (up to 5\%) than the difference between reconstructions and model function, suggesting reliability of the rolling iteration for sudden changes in the emissivity profile.

\section{Summary}

The contribution deals with optimization of the Tikhonov regularization constrained by minimum Fisher information for SXR tomography by means of symmetrization of calculation of the Fisher information, choice of border conditions, and adaptation of the algorithm for real-time tomography. Symmetrization of the Fisher information was shown to suppress the artifacts that can occur in reconstructed emissivity. Border conditions in the form of the exclusion of pixels outside the chamber and requirement of zero emissivity at chamber's pixels led to faster reconstruction with a more reliable solution. In the case of fast changes in plasma emissivity, the algorithm for real-time tomography typically needs several frames to approach the correct solution, but it still provides a reliable solution as the discrepancy between the real-time and standard algorithm is not high (below 10\%).
Acknowledgments. This project has received funding from the European Union's Horizon 2020 Research and Innovation programme under grant agreement no. 633053. The views and opinions expressed herein do not necessarily reflect those of the European Commission. This work was supported by the MSMT projects nos. LM2011021, LG14002, and 8D15001.

\section{References}

1. Panek, R., Bilková, P., Fuchs, V., Hron, M., Chraska, P., Stockel, J., Urban, J., Weinzettl, V., Zajac, J., \& Zacek, F. (2006). Reinstallation of the COMPASS-D tokamak in IPP ASCR. Czech. J. Phys., 56(Suppl. 2), B125-B137. DOI: 10.1007/s10582-006-0188-1.

2. Weinzettl, V., Naydenkova, D. I., Sestak, D., Vlcek, J., Mlynar, J., Melich, R., Jares, D., Malot, J., Sarychev, D., \& Igochine, V. (2010). Design of multi-range tomographic system for transport studies in tokamak plasmas. Nucl. Instrum. Methods Phys. Res. Sect. AAccel. Spectrom. Dect. Assoc. Equip., 623, 806-808. DOI: 10.1016/j.nima.2010.04.010.

3. Anton, M., Weisen, H., Dutch, M. J., Linden, W., Buhlmann, F., Chavan, R., Marletaz, B., Marmillod, P., \& Paris, P. (1996). X-ray tomography on the TCV tokamak. Plasma Phys. Control. Fusion, 38, 1849-1878. DOI: 10.1088/0741-3335/38/11/001.

4. Odstrcil, M., Mlynar, J., Odstrcil, T., Alper, B., Murari, A., \& JET EFDA Contributors. (2012). Modern numerical methods for plasma tomography optimization. Nucl. Instrum. Methods Phys. Res. Sect. A-Accel. Spectrom. Dect. Assoc. Equip., 686, 156-161.

5. Mazon, D., Vezinet, D., Pacella, D., Moreau, D., Gabelieri, L., Romano, A., Malard, P., Mlynar, J., Masset, R., \& Lotte, P. (2012). Soft x-ray tomography for real-time applications: present status at Tore Supra and possible future developments. Rev. Sci. Instrum., 83(6), 063505. DOI: 10.1063/1.4730044.

6. Loffelmann, V., Mlynar, J., Imrisek, M., Mazon, D., Jardin, A., Weinzettl, V., \& Hron, M. (2016). Minimum Fisher Tikhonov regularization adapted to real-time tomography. Fusion Sci. Technol., 69(2), 505-513.

7. Ingesson, L. C., Alper, B., Peterson, B. J., \& Vallet, J. -C. (2008). Tomography diagnostics: Bolometry and soft X-ray detection. Fusion Sci. Technol., 53(2), 528-576. 